\title{
Antiviral Therapy Improves Hepatocellular Cancer Survival
}

\author{
Ngan Nguyen, DO; Kruti Patel, DO; Anna Carson Uhelski, MD; Bradford Waters, MD; and Alva Weir, MD
}

Background: Chronic hepatitis $\mathrm{C}$ virus (HCV) infection is a common risk factor for hepatocellular cancer (HCC). Patients with HCV infection are at a higher risk of developing HCC because the virus induces fibrosis in the liver, which may lead to cirrhosis. Early treatment of HCV and achieving a sustained virologic response (SVR) may lead to decreased incidence and mortality associated with HCC.

Methods: We performed a retrospective review of patients at the Memphis Veterans Affairs Medical Center (VAMC) in Tennessee from November 2008 to March 2019 to determine whether treatment of HCV infection makes a difference in overall survival (OS) among patients who develop HCC. Patients were treated with an interferon-based regimen or direct-acting antiviral agents (DAAs). Among the patients with $\mathrm{HCV}$ infection who were treated, we identified those who did achieve or did not achieve SVR.

Results: We identified 111 patients with HCV and HCC; 68 were treated for HCV infection. Forty-eight patients received DAA and 20 patients received an interferon-based regimen and 51 achieved SVR. In a multivariate analysis accounting for severity of liver disease, treated patients had an improved 5-year OS rate, median 1338 days $(95 \% \mathrm{Cl}$, 966-3202) when compared with untreated patients whose median OS was 452 days $(95 \% \mathrm{Cl}, 242-853)(P=.0005)$. The treatment group had a longer median progression-free survival (PFS) than did the nontreatment group (460 days [95\% $\mathrm{Cl}, 294-726$ ] vs 286 days [95\% Cl, 205-405], $P=.04$ ). $\mathrm{Pa}-$ tients with SVR had an increased 5-year OS compared with patients without SVR (median 1973 days [95\% Cl, 1222-NA] vs 470 days [95\% Cl, 242-853], $P<.001)$. HCV treatment type (interferon vs DAA) was not found to be associated with either OS or PFS, regardless of time period. Advanced liver disease stage as characterized by a high model for end-stage liver disease (MELD) score (>10) or high Child-Pugh score (B or $\mathrm{C}$ ) was associated with worse survival outcome.

Conclusions: A retrospective analysis of patients with $\mathrm{HCV}$ infection and HCC confirms that treatment of HCV infection leads to OS benefit among patients with HCC. We further demonstrate that patients with HCV infection who achieve SVR have an OS benefit over patients unable to achieve SVR. The type of treatment, DAA vs an interferon-based regimen, did not show a significant survival benefit.

\section{Author affiliations}

can be found at the end of the article. Correspondence: Alva Weir

(alva.weir@va.gov)

Fed Pract. 2021;38(suppl 3). Published online

September 3. doi:10.12788/fp.0165
$\mathrm{H}$ epatocellular cancer $(\mathrm{HCC})$ is the most common type of hepatic cancers, accounting for $65 \%$ of all hepatic cancers. ${ }^{1}$ Among all cancers, $\mathrm{HCC}$ is one of the fastest growing causes of death in the United States, and the rate of new HCC cases are on the rise over several decades. ${ }^{2}$ There are many risk factors leading to HCC, including alcohol use, obesity, and smoking. Infection with hepatitis $C$ virus (HCV) poses a significant risk. ${ }^{1}$

The pathogenesis of HCV-induced carcinogenesis is mediated by a unique host-induced immunologic response. Viral replication induces production of inflammatory factors, such as tumor necrosis factor (TNF- $\alpha$ ), interferon (IFN), and oxidative stress on hepatocytes, resulting in cell injury, death, and regeneration. Repetitive cycles of cellular death and regeneration induce fibrosis, which may lead to cirrhosis. ${ }^{3}$ Hence, early treatment of HCV infection and achieving sustained virologic response (SVR) may lead to decreased incidence and mortality associated with HCC.

Treatment of HCV infection has become more effective with the development of direct-acting antivirals (DAAs) leading to SVR in $>90 \%$ of patients compared with 40 to $50 \%$ with IFN-based treatment. ${ }^{4,5}$ DAAs have been proved safe and highly effective in eradicating HCV infection even in patients with advanced liver disease with decompensated cirrhosis. ${ }^{6}$ Although achieving SVR indicates a complete cure from chronic HCV infection, several studies have shown subsequent risk of developing HCC persists even after successful HCV treatment. ${ }^{7-9}$ Some studies show that using DAAs to achieve SVR in patients with $\mathrm{HCV}$ infection leads to a decreased relative risk of HCC development compared with patients who do not receive treatment. ${ }^{10-12}$ But data on HCC risk following DAA-induced SVR vs IFNinduced SVR are somewhat conflicting.

Much of the information regarding the association between SVR and HCC has been gleaned from large data banks without accounting for individual patient characteristics that can be obtained through full chart review. Due to small sample sizes in many chart review studies, the impact that SVR from DAA therapy has on the progression and severity of HCC is not entirely clear. The aim of our study is to evaluate the effect of HCV treatment and SVR status on overall survival (OS) in patients with HCC. Second, we aim to compare survival benefits, if any exist, among the 2 major HCV treatment modalities (IFN vs DAA).

\section{METHODS}

We performed a retrospective review of patients at Memphis Veterans Affairs Medical Center 
(VAMC) in Tennessee to determine whether treatment for HCV infection in general, and achieving SVR in particular, makes a difference in progression, recurrence, or OS among patients with HCV infection who develop HCC. We identified 111 patients with a diagnosis of both HCV and new or recurrent $\mathrm{HCC}$ lesions from November 2008 to March 2019 (Table 1). We divided these patients based on their HCV treatment status, SVR status, and treatment types (IFN vs DAA).

The inclusion criteria were patients aged $>18$ years treated at the Memphis VAMC who have HCV infection and developed HCC. Exclusion criteria were patients who developed HCC from other causes such as alcoholic steatohepatitis, hepatitis $B$ virus infection, hemochromatosis, patients without $\mathrm{HCV}$ infection, and patients who were not established at the Memphis VAMC. This protocol was approved by the Memphis VAMC Institutional Review Board.

$\mathrm{HCC}$ diagnosis was determined using International Classification of Diseases codes (9th revision: 155 and 155.2; 10th revision: CD 22 and 22.9). We also used records of multidisciplinary gastrointestinal malignancy tumor conferences to identify patient who had been diagnosed and treated for HCV infection. We identified patients who were treated with DAA vs IFN as well as patients who had achieved SVR (classified as having negative HCV RNA tests at the end of DAA treatment). We were unable to evaluate BarceIona Clinic Liver Cancer staging since this required documented performance status that was not available in many patient records. We selected cases consistent with both treatment for HCV infection and subsequent development of HCC. Patient data included age; OS time; HIV status HCV genotype; time and status of progression to HCC; type and duration of treatment; and alcohol, tobacco, and drug use. Disease status was measured using the Model for EndStage Liver Disease (MELD) score (Table 2), Milan criteria (Table 3), and Child-Pugh score (Table 4).

\section{Statistical Analysis}

OS was measured from the date of HCC diagnosis to the date of death or last follow-up. Progression-free survival (PFS) was defined from the date of $\mathrm{HCC}$ treatment initiation to the date of first HCC recurrence. We compared survival data for the SVR and non-SVR subgroups, the HCV treatment vs non-HCV treatment subgroups, and the IFN therapy vs DAA therapy
TABLE 1 Characteristics of Patients With HCV and HCC

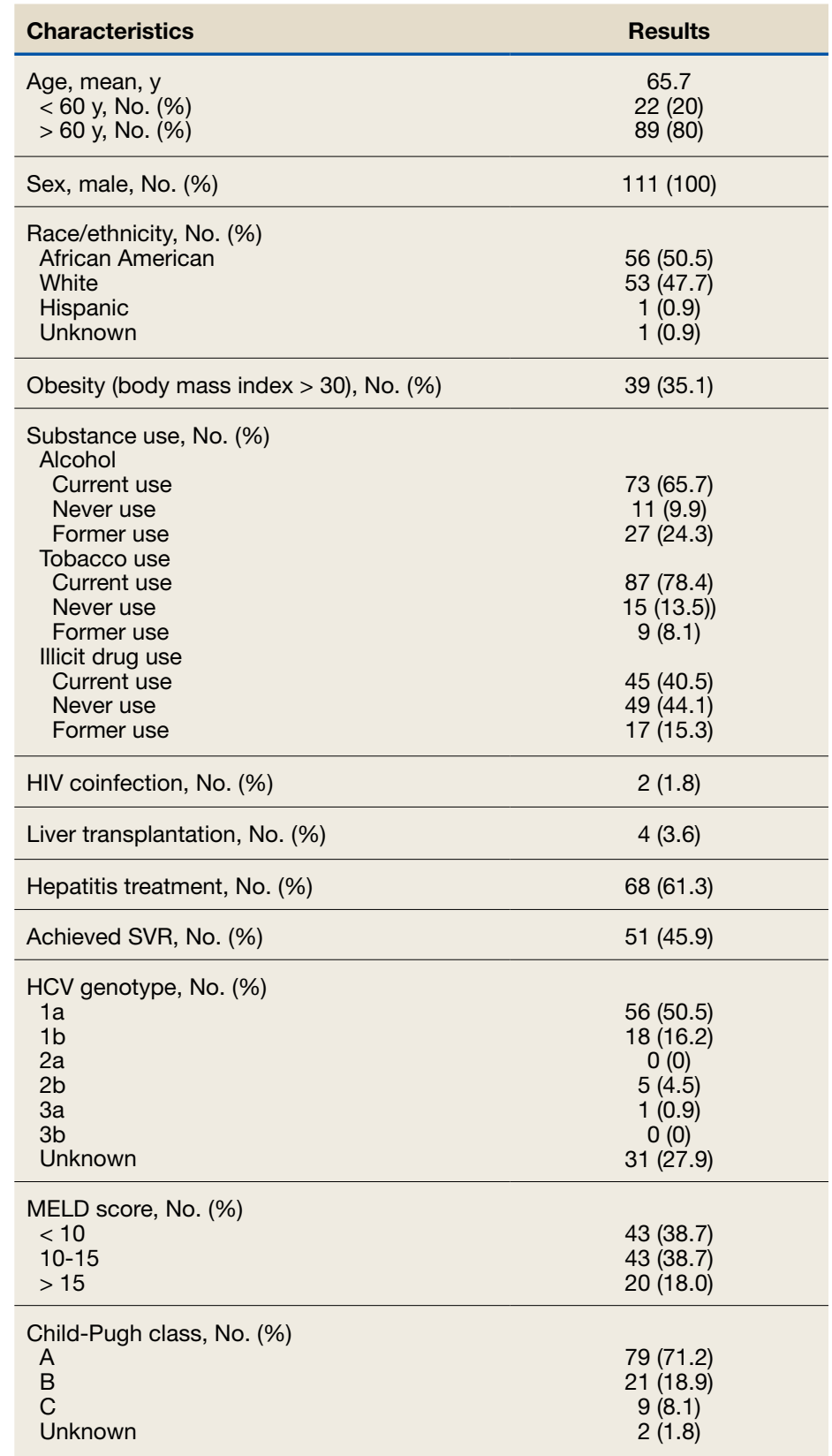

Abbreviations: HCC, hepatocellular cancer; HCV, hepatitis C virus; MELD, Model for End-Stage Liver Disease; SVR, sustained virologic response.

subgroups, using the Kaplan-Meier method. The differences between subgroups were assessed using a log-rank test. Multivariate analysis using Cox proportional hazards regression model was used to identify factors that had significant impact on OS. Those factors included age; race; alcohol, tobacco, and illicit drug use; SVR status; HCV treatment status; IFN-based regimen vs DAA; MELD, and Child-Pugh scores. The 
TABLE 2 Model for End-Stage Liver Disease Scores ${ }^{24, a}$

\begin{tabular}{cc} 
Scores & 90-Day Mortality, \% \\
\hline$\geq 40$ & 71.3 \\
$30-39$ & 52.6 \\
$20-29$ & 19.6 \\
$10-19$ & 6 \\
$\leq 9$ & 1.9
\end{tabular}

aScore calculated using the formula $9.57 \times$ loge (creatinine) $+3.78 \times$ loge (total bilirubin) $+11.2 \times$ loge (international normalized ratio) +6.43 .

\section{TABLE 3 Milan Criteria ${ }^{\mathrm{a}}$}

- Single tumor $\leq 5 \mathrm{~cm}$ or 3 lesions $\leq 3 \mathrm{~cm}$ each

- No evidence of vascular invasion

- No evidence of extrahepatic metastatic disease

aAll criteria must be met.

TABLE 4 Child-Pugh Classification for Severity of Cirrhosis

\begin{tabular}{|c|c|}
\hline Clinical Criteria & Points \\
\hline \multicolumn{2}{|l|}{ Encephalopathy } \\
\hline None & 1 \\
\hline Mild to moderate (grade 1 or 2) & 2 \\
\hline Severe (grade 3 or 4 ) & 3 \\
\hline \multicolumn{2}{|l|}{ Ascites } \\
\hline None & 1 \\
\hline Mild to moderate (diuretic response) & 2 \\
\hline Severe (diuretic refractory) & 3 \\
\hline \multicolumn{2}{|l|}{ Bilirubin, mg/dL } \\
\hline$<2$ & 1 \\
\hline $2-3$ & 2 \\
\hline$>3$ & 3 \\
\hline \multicolumn{2}{|l|}{ Albumin, g/dL } \\
\hline$>3.5$ & 1 \\
\hline $2.8-3.5$ & 2 \\
\hline$<2.8$ & 3 \\
\hline \multicolumn{2}{|l|}{ Prothrombin time } \\
\hline \multicolumn{2}{|l|}{ Prolonged, sec } \\
\hline$<4$ & 1 \\
\hline $4-6$ & 2 \\
\hline$>6$ & 3 \\
\hline \multicolumn{2}{|l|}{ International normalized ratio } \\
\hline$<1.7$ & 1 \\
\hline $1.7-2.3$ & 2 \\
\hline$>2.3$ & 3 \\
\hline \multicolumn{2}{|l|}{ Class scoring (severity) } \\
\hline \multicolumn{2}{|l|}{ A: $5-6$ points (least) } \\
\hline \multicolumn{2}{|l|}{ B: $7-9$ points (moderate) } \\
\hline C: $10-15$ points (highest) & \\
\hline
\end{tabular}

results were expressed as hazard ratios (HRs) and $95 \% \mathrm{Cl}$. Calculations were made using Statistical Analysis SAS and IBM SPSS software.

\section{RESULTS}

The study included 111 patients. The mean age was 65.7 years; all were male and half of were Black patients. The gender imbalance was due to the predominantly male patient population at Memphis VAMC. Among 111 patients with HCV infection and HCC, 68 patients were treated for $\mathrm{HCV}$ infection and had significantly improved OS and PFS compared with the nontreatment group. The median 5-year OS was 44.6 months (95\% $\mathrm{Cl}, 966-3202)$ in the treated HCV infection group compared with 15.1 months in the untreated HCV infection group with a Wilcoxon $P=.0005$ (Figure 1). Similarly, patients treated for HCV infection had a significantly better 5 -year PFS of 15.3 months $(95 \% \mathrm{Cl}, 294-726)$ compared with the nontreatment group 9.5 months $(95 \% \mathrm{Cl}$, 205-405) with a Wilcoxon $P=.04$ (Figure 2).

Among 68 patients treated for HCV infection, 51 achieved SVR, and 34 achieved SVR after the diagnosis of HCC. Patients who achieved SVR had an improved 5-year OS when compared with patients who did not achieve SVR (median 65.8 months [95\% Cl, 1222-NA] vs 15.7 months [95\% Cl, 242-853], Wilcoxon $P<.001$ ) (Figure 3). Similarly, patients with SVR had improved 5-year PFS when compared with the non-SVR group (median 20.5 months [95\% Cl, 431-914] vs 8.9 months [95\% Cl, 191-340], Wilcoxon $P=.007$ (Figure 4). Achievement of SVR after HCC diagnosis suggests a significantly improved OS (HR 0.37) compared with achievement prior to HCC diagnosis (HR, 0.65; 95\% Cl, 0.23-1.82, $P=.41$ )

Multivariate Cox regression was used to determine factors with significant survival impact. Advanced age at diagnosis (aged $\geq$ 65 years) (HR, 0.53; 95\% Cl, 0.320-0.880; $P=.01)$, SVR status (HR, $0.33 ; 95 \% \mathrm{Cl}$, 0.190-0.587; $P<.001)$, achieving SVR after $\mathrm{HCC}$ diagnosis (HR, 0.37; $95 \% \mathrm{Cl}, 0.20-$ $0.71 ; P=.002)$, low MELD score $(<10)$ (HR, 0.49 ; $95 \% \mathrm{Cl}, 0.30-0.80 ; P=.004$ ) and low Child-Pugh score (class A) (HR, 0.39; $95 \% \mathrm{Cl}$, $0.24-0.64 ; P=.001$ ) have a significant positive impact on OS. Survival was not significantly influenced by race, tobacco, drug use, HIV or cirrhosis status, or HCV treatment type. In addition, higher Child-Pugh class ( $\mathrm{B}$ or $\mathrm{C}$ ), higher MELD score ( $>10)$, and younger age at 
FIGURE 1 HCV Treated vs Not-Treated 5-Year OS

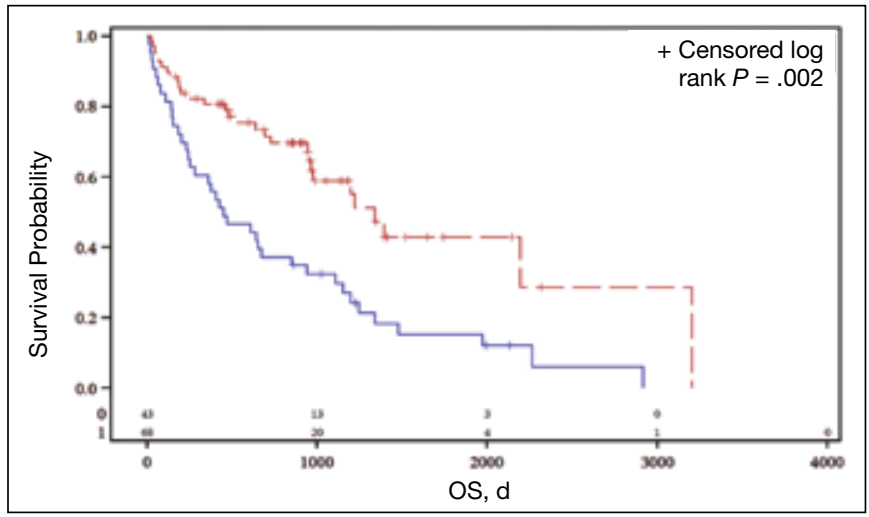

FIGURE 3 SVR vs No SVR 5-Year OS

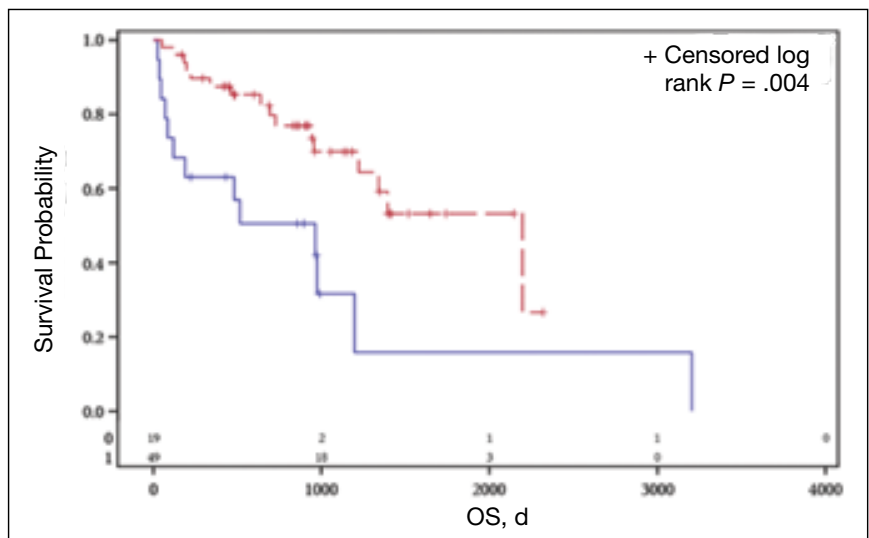

FIGURE 2 HCV Treated vs Not-Treated 5-Year PFS

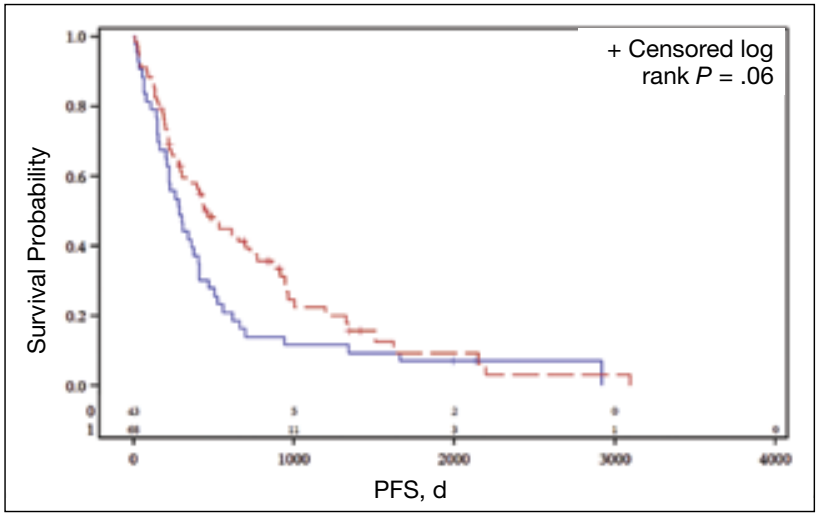

FIGURE 4 SVR vs No SVR 5-Year PFS

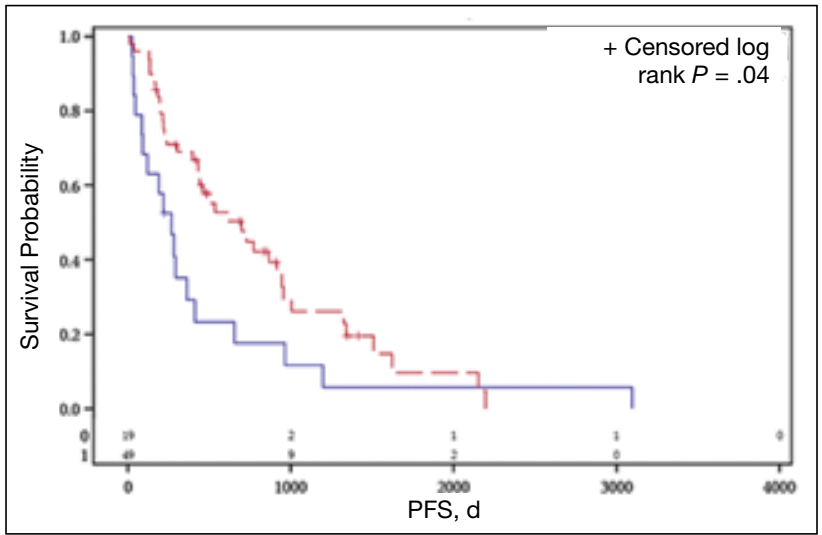

Abbreviations: Abbreviations: HCV, hepatitis C virus; OS overall survival; PFS, progression-free survival; SVR, sustained virologic response.

diagnosis (<65 years) have a negative impact on survival outcome (Table 5).

\section{DISCUSSION}

The survival benefit of HCV eradication and achieving SVR status has been well established in patients with $\mathrm{HCC} .{ }^{13}$ In a retrospective cohort study of 250 patients with HCV infection who had received curative treatment for $\mathrm{HCC}$, multivariate analysis demonstrated that achieving SVR is an independent predictor of OS. ${ }^{14}$ The 3-year and 5 -year OS rates were $97 \%$ and $94 \%$ for the SVR group, and $91 \%$ and $60 \%$ for the non-SVR group, respectively $(P<.001)$. Similarly, according to Sou and colleagues, of 122 patients with HCVrelated HCC, patients with SVR had longer OS than patients with no SVR $(P=.04) .{ }^{15}$ One of the hypotheses that could explain the survival benefit in patients who achieved SVR is the effect of achieving SVR in reducing persistent liver inflammation and associated liver mortality, and therefore lowering risks of complication in patients with HCC. ${ }^{16}$ In our study, multivariate analysis shows that achieving SVR is associated with significant improved OS (HR, 0.33). In contrast, patients with HCC who have not achieved SVR are associated with worse survival (HR, 3.24). This finding supports early treatment of HCV to obtain SVR in HCV-related patients with HCC, even after development of HCC.

Among 68 patients treated for HCV infection, 45 patients were treated after HCC diagnosis, and 34 patients achieved SVR after HCC diagnosis. The average time between HCV infection treatment after HCC diagnosis was 6 months. Our data suggested that achievement of SVR after $\mathrm{HCC}$ diagnosis suggests an improved OS (HR, 0.37) compared with achievement prior to HCC diagnosis (HR, 0.65; 95\% Cl,0.23-1.82; $P=.41)$. This lack of statistical significance is likely due to small sample size of patients achieving SVR prior to HCC diagnosis. Our results are consistent with the findings regarding the efficacy and timing of DAA treatment in patients with active HCC. According to Singal and colleagues, achieving SVR after DAA therapy may result in improved liver 
TABLE 5 Multivariate Survival Analysis

\begin{tabular}{|c|c|c|}
\hline Variables & Hazard Ratio $(95 \% \mathrm{Cl})$ & $P$ value \\
\hline $\begin{array}{l}\text { Age, y } \\
\geq 65 \\
<65\end{array}$ & $\begin{array}{l}0.53(0.32-0.88) \\
1.89(1.14-3.13)\end{array}$ & $\begin{array}{l}.01 \\
.01\end{array}$ \\
\hline $\begin{array}{l}\text { Race } \\
\text { African American } \\
\text { White }\end{array}$ & $\begin{array}{c}1.39(0.85-2.28) \\
1.44(0.19-10.73)\end{array}$ & $\begin{array}{l}.19 \\
.72\end{array}$ \\
\hline $\begin{array}{l}\text { Substance use } \\
\text { Alcohol } \\
\text { Tobacco } \\
\text { Illicit drug }\end{array}$ & $\begin{array}{l}1.66(0.91-3.00) \\
1.54(0.66-3.61) \\
1.17(0.66-2.07)\end{array}$ & $\begin{array}{l}.10 \\
.32 \\
.60\end{array}$ \\
\hline $\begin{array}{l}\text { Comorbid conditions } \\
\text { HIV } \\
\text { Liver cirrhosis }\end{array}$ & $\begin{array}{l}0.62(0.08-4.69) \\
2.25(0.95-5.31)\end{array}$ & $\begin{array}{l}.64 \\
.07\end{array}$ \\
\hline $\begin{array}{l}\text { Sustained virologic response } \\
\text { Yes } \\
\text { No } \\
\text { Before HCC diagnosis } \\
\text { After HCC diagnosis }\end{array}$ & $\begin{array}{l}0.33(0.19-0.59) \\
3.24(1.85-5.69) \\
0.65(0.23-1.82) \\
0.37(0.20-0.71)\end{array}$ & $\begin{array}{l}<.001 \\
<.001 \\
.41 \\
.002\end{array}$ \\
\hline $\begin{array}{l}\text { Treatment types } \\
\text { Direct-acting antiviral } \\
\text { Interferon-based regimen }\end{array}$ & $\begin{array}{l}0.61(0.30-1.26 \\
1.01(0.22-4.69)\end{array}$ & $\begin{array}{l}.18 \\
.11\end{array}$ \\
\hline $\begin{array}{l}\text { Model for End-Stage Liver } \\
\text { Disease score } \\
\leq 10 \\
>10\end{array}$ & $\begin{array}{c}0.49(0.30-0.80) \\
2.202(1.36-3.58)\end{array}$ & $\begin{array}{l}.004 \\
.001\end{array}$ \\
\hline $\begin{array}{l}\text { Child-Pugh class } \\
\text { A } \\
\text { B or C }\end{array}$ & $\begin{array}{l}0.39(0.24-0.64) \\
2.57(1.56-4.21)\end{array}$ & $\begin{array}{l}.001 \\
.001\end{array}$ \\
\hline
\end{tabular}

Abbreviation: HCC, hepatocellular carcinoma.

function and facilitate additional HCC-directed therapy, which potentially improves survival..$^{17-19}$

Nagaoki and colleagues found that there was no significant difference in OS in patients with HCC between the DAA and IFN groups. According to the study, the 3-year and 5-year OS rates were $96 \%$ and $96 \%$ for DAA patients and $93 \%$ and $73 \%$ for IFN patients, respectively $(P=.16) .^{14}$ This finding is consistent with the results of our study. HCV treatment type (IFN vs DAA) was not found to be associated with either OS or PFS time, regardless of time period.

A higher MELD score $(>10)$ and a higher Child-Pugh class (B or C) score are associated with worse survival outcome regardless of SVR status. While patients with a low MELD score $(\leq 10)$ have a better survival rate (HR 0.49), a higher MELD score has a significantly higher $H R$ and therefore worse survival outcomes (HR, 2.20). Similarly, patients with Child-Pugh A (HR, 0.39) have a better survival outcome compared with those patients with Child-Pugh class $B$ or $C(H R, 2.57)$. This finding is consistent with re- sults of multiple studies indicating that advanced liver disease, as measured by a high MELD score and Child-Pugh class score, can be used to predict the survival outcome in patients with HCVrelated HCC. .0-22 $^{2}$

Unlike other studies that look at a single prognostic variable, our study evaluated prognostic impacts of multiple variables (age, SVR status, the order of SVR in relation to HCC development, HCV treatment type, MELD score and ChildPugh class) in patients with HCC. The study included patients treated for HCV after development of $\mathrm{HCC}$ along with other multiple variables leading to OS benefit. It is one of the only studies in the United States that compared 5-year OS and PFS among patients with HCC treated for HCV and achieved SVR. The studies by Nagaoki and colleagues and Sou and colleagues were conducted in Japan, and some of their subset analyses were univariate. Among our study population of veterans, $50 \%$ were African American patients, suggesting that they may have similar OS benefit when compared to White patients with $\mathrm{HCC}$ and HCV treatment.

\section{Limitations}

Our findings were limited in that our study population is too small to conduct further subset analysis that would allow statistical significance of those subsets, such as the suggested benefit of SVR in patients who presented with HCC after antiviral therapy. Another limitation is the all-male population, likely a result of the older veteran population at the Memphis VAMC. The mean age at diagnosis was 65 years, which is slightly higher than the general population. Compared to the SEER database, HCC is most frequently diagnosed among people aged 55 to 64 years. ${ }^{23}$ The age difference was likely due to our aging veteran population.

Further studies are needed to determine the significance of SVR on HCC recurrence and treatment. Immunotherapy is now first-line treatment for patients with local advanced HCC. All the immunotherapy studies excluded patients with active HCV infection. Hence, we need more data on HCV treatment timing among patients scheduled to start treatment with immunotherapy.

\section{CONCLUSIONS}

In a population of older veterans, treatment of HCV infection leads to OS benefit among patients with HCC. In addition, patients with HCV infection who achieve SVR have an OS 
benefit over patients unable to achieve SVR. The type of treatment, DAA vs IFN-based regimen, did not show significant survival benefit.

\section{Author Affiliations}

Bradford Waters is a Gastroenterologist in the Gastroenterology \& Hepatology Department; and Alva Weir is a Hematologist Oncologist, Section Chief Hematology/Oncology, both at the Memphis Veteran Affairs Medical Center in Tennessee. Anna Carson Uhelski is a Medicine Resident Physician at Johns Hopkins Osler in Baltimore Maryland. Bradford Waters and Alva Weir are Professors; and Ngan Nguyen and Kruti Patel are Hematology Oncology Fellows, all at the University of Tennessee Health Science Center, in Memphis.

\section{Author disclosures}

The authors report no actual or potential conflicts of interest with regard to this article.

\section{Disclaimer}

The opinions expressed herein are those of the authors and do not necessarily reflect those of Federal Practitioner, Frontline Medical Communications Inc., the US Government, or any of its agencies. This article may discuss unlabeled or investigational use of certain drugs. Please review the complete prescribing information for specific drugs or drug combinations-including indications, contraindications, warnings, and adverse effects-before administering pharmacologic therapy to patients.

\section{References}

1. Ghouri YA, Mian I, Rowe JH. Review of hepatocellular carcinoma: epidemiology, etiology, and carcinogenesis. J Carcinog. 2017;16:1. Published 2017 May 29. doi:10.4103/jcar.JCar_9_16

2. Bray F, Ferlay J, Soerjomataram I, Siegel RL, Torre LA, Jemal A. Global cancer statistics 2018: GLOBOCAN estimates of incidence and mortality worldwide for 36 cancers in 185 countries. CA Cancer J Clin. 2018;68(6):394-424. doi:10.3322/caac.21492

3. Farazi PA, DePinho RA. Hepatocellular carcinoma pathogenesis: from genes to environment. Nat Rev Cancer. 2006;6(9):674-687. doi:10.1038/nrc1934

4. Falade-Nwulia O, Suarez-Cuervo C, Nelson DR, Fried MW, Segal JB, Sulkowski MS. Oral direct-acting agent therapy for hepatitis c virus infection: a systematic review. Ann Intern Med. 2017;166(9):637-648. doi:10.7326/M16-2575

5. Kouris G, Hydery T, Greenwood BC, et al. Effectiveness of Ledipasvir/Sofosbuvir and predictors of treatment failure in members with hepatitis $C$ genotype 1 infection: a retrospective cohort study in a medicaid population. J Manag Care Spec Pharm. 2018;24(7):591-597. doi:10.18553/jmcp.2018.24.7.591

6. Jacobson IM, Lawitz E, Kwo PY, et al. Safety and efficacy of elbasvir/grazoprevir in patients with hepatitis $C$ virus infection and compensated cirrhosis: an integrated analysis. Gastroenterology. 2017;152(6):1372-1382.e2. doi:10.1053/j.gastro.2017.01.050

7. Nahon P, Layese R, Bourcier V, et al. Incidence of hepatocellular carcinoma after direct antiviral therapy for $\mathrm{HCV}$ in patients with cirrhosis included in surveillance programs. Gastroenterology. 2018;155(5):1436-1450.e6. doi:10.1053/j.gastro.2018.07.01510.

8. Innes H, Barclay ST, Hayes PC, et al. The risk of hepatocellular carcinoma in cirrhotic patients with hepatitis $C$ and sustained viral response: role of the treatment regimen. J Hepatol. 2018;68(4):646-654. doi:10.1016/j.jhep.2017.10.033

9. Romano A, Angeli P, Piovesan S, et al. Newly diagnosed hepatocellular carcinoma in patients with advanced hepatitis $C$ treated with DAAs: a prospective population study. $J$ Hepatol. 2018;69(2):345-352. doi:10.1016/j.jhep.2018.03.009

10. Kanwal F, Kramer J, Asch SM, Chayanupatkul M, Cao Y, El-Serag HB. Risk of hepatocellular cancer in HCV patients treated with direct-acting antiviral agents. Gastroenterology. 2017;153(4):996-1005.e1. doi:10.1053/j.gastro.2017.06.0122

11. Singh S, Nautiyal A, Loke YK. Oral direct-acting antivirals and the incidence or recurrence of hepatocellular carcinoma: a systematic review and metaanalysis. Frontline Gastroenterol. 2018;9(4):262-270. doi:10.1136/flgastro-2018-101017

12. Kuftinec G, Loehfelm T, Corwin M, et al. De novo hepatocellular carcinoma occurrence in hepatitis $\mathrm{C}$ cirrhotics treated with direct-acting antiviral agents. Hepat Oncol. 2018;5(1):HEP06. Published 2018 Jul 25. doi:10.2217/hep-2018-00033

13. Morgan RL, Baack B, Smith BD, Yartel A, Pitasi M, FalckYtter $Y$. Eradication of hepatitis $C$ virus infection and the development of hepatocellular carcinoma: a meta-analysis of observational studies. Ann Intern Med. 2013;158(5 Pt 1):329337. doi:10.7326/0003-4819-158-5-201303050-00005

14. Nagaoki $Y$, Imamura $M$, Nishida $Y$, et al. The impact of interferon-free direct-acting antivirals on clinical outcome after curative treatment for hepatitis $\mathrm{C}$ virus-associated hepatocellular carcinoma: comparison with interferon-based therapy. J Med Virol. 2019;91(4):650-658. doi:10.1002/jmv.25352

15. Sou FM, Wu CK, Chang KC, et al. Clinical characteristics and prognosis of HCC occurrence after antiviral therapy for HCV patients between sustained and non-sustained responders. J Formos Med Assoc. 2019;118(1 Pt 3):504-513. doi:10.1016/j.jma.2018.10.017

16. Roche B, Coilly A, Duclos-Vallee JC, Samuel D. The impact of treatment of hepatitis $C$ with DAAs on the occurrence of HCC. Liver Int. 2018;38(suppl 1):139-145. doi:10.1111/liv.13659

17. Singal AG, Lim JK, Kanwal F. AGA clinical practice update on interaction between oral direct-acting antivirals for chronic hepatitis $\mathrm{C}$ infection and hepatocellular carcinoma: expert review. Gastroenterology. 2019;156(8):2149-2157. doi:10.1053/j.gastro.2019.02.046

18. Toyoda H, Kumada T, Hayashi K, et al. Characteristics and prognosis of hepatocellular carcinoma detected in sustained responders to interferon therapy for chronic hepatitis C. Cancer Detect Prev. 2003;27(6):498-502. doi:10.1016/j.cdp.2003.09.007

19. Okamura $Y$, Sugiura $T$, Ito $T$, et al. The achievement of a sustained virological response either before or after hepatectomy improves the prognosis of patients with primary hepatitis C virus-related hepatocellular carcinoma. Ann Surg Oncol. 2019; 26(13):4566-4575. doi:10.1245/s10434-019-07911-w

20. Wray CJ, Harvin JA, Silberfein EJ, Ko TC, Kao LS. Pilot prognostic model of extremely poor survival among high-risk hepatocellular carcinoma patients. Cancer. 2012;118(24):6118-6125. doi:10.1002/cncr.27649

21. Kim JH, Kim JH, Choi JH, et al. Value of the model for endstage liver disease for predicting survival in hepatocellular carcinoma patients treated with transarterial chemoembolization. Scand J Gastroenterol. 2009;44(3):346-357. doi:10.1080/00365520802530838

22. Vogeler M, Mohr I, Pfeiffenberger J, et al. Applicability of scoring systems predicting outcome of transarterial chemoembolization for hepatocellular carcinoma. J Cancer Res Clin Oncol. 2020;146(4):1033-1050. doi:10.1007/s00432-020-03135-8

23. National Institutes of Health, Surveillance, Epidemiology, and End Results. Cancer stat facts: cancer of the liver and intrahepatic bile duct. Accessed July 15, 2021. https://seer .cancer.gov/statfacts/html/livibd.html

24. Singal AK, Kamath PS. Model for End-stage Liver Disease. J Clin Exp Hepatol. 2013;3(1):50-60. doi:10.1016/j.jceh.2012.11.002 